\title{
RIMAPS Prediction of Etch Pit Patterns
}

\author{
Néstor O. Fuentes ${ }^{1,2}$ and Eduardo A. Favret ${ }^{2,3,4}$ \\ ${ }^{1}$ Comisión Nacional de Energía Atómica (CNEA), Gerencia de Área Seguridad Nuclear y \\ Ambiente, PNGRR. Av. del Libertador 8250. C1429BNP. Ciudad Autónoma de Buenos Aires \\ Argentina \\ 2 Instituto de Tecnología "Prof. Jorge A. Sabato", Universidad Nacional de Gral. San Martín. Av. \\ Gral. Paz 1499. B1650KNA. San Martín. Buenos Aires. Argentina \\ ${ }^{3}$ Instituto Nacional de Tecnología Agropecuaria (INTA), Instituto de Suelos. De Los Reseros y N. \\ Repetto s/n. 1686. Hurlingham. Buenos Aires. Argentina \\ ${ }^{4}$ Consejo Nacional de Investigaciones Científicas y Técnicas (CONICET), Av. Rivadavia 1917. \\ C1033AAJ. Ciudad Autónoma de Buenos Aires. Argentina
}

During the last decade, RIMAPS (Rotated Image with Maximum Average Power Spectrum) technique has been extensively used finding the main directions of any surface topography and describing it by simple geometrical figures [1]. This technique has already been applied to the study of metallic surfaces [2], biological samples [3][4] and worked materials on used edges of lithic artifacts [5][6]. In this work, RIMAPS technique is extended to predict the topographic pattern produced by etching metallic surfaces, using aluminum to give examples of RIMAPS prediction capability. The aluminum samples were annealed at a temperature of $550^{\circ} \mathrm{C}$ during 30 hours. Then, the surfaces were mechanically ground with emery paper down to 1000 grit. A solution of butyl cellosolve $90 \%$ - perchloric acid $10 \%$ was used for the electrolytical polishing. The etching was carried out at room temperature using the Tucker reagent. From a single etch pitting figure (Fig. 1) the corresponding sketch is obtained to represent the characteristic simple geometrical shape of etching process (Fig. 2). Then, RIMAPS spectrum from the sketch is obtained (Fig. 3). When the whole topographic pattern on aluminum surface (Fig. 4) is analyzed using RIMAPS (Fig. 5), comparison can be made between the spectra of the single sketch and the etching topographic pattern (Fig. 6). The angular positions of the maxima of both RIMAPS spectra are coincident, showing that the arrangement of numerous etch pitting figures does not modify the RIMAPS spectrum of one etch pit. It is well known that RIMAPS determines not only the orientations of topographic patterns, but also gives notice of the existence of surface deformation with the appearance of peaks denoting secondary directions. The remarkable coincidence of peaks position between the single figure sketch and the experimental aluminum surface, turns out to light the fact that RIMAPS can predict the real pattern to be obtained after etching and emphasizes the potential of this technique either for characterization and prediction of any surface topographic pattern.

\section{References}

[1] N. O. Fuentes and E. A. Favret, Journal of Microscopy. 206 (2002) 72-83.

[2] E. A. Favret, N. O. Fuentes and F. Yu, Applied Surface Science. 230 (2004) 60-72.

[3] E. A. Favret and N. O. Fuentes, Microscopy Today. 12 (2004) 24-26.

[4] E. A. Favret et al., Microscopy Research and Technique. 69 (2006) 684-688.

[5] E. A. Favret, N. O. Fuentes and M. R. Alvarez, Micros. Microanal. 10(Suppl 2) (2004) 968-969.

[6] M. Álvarez, N. O. Fuentes, E. A. Favret, M. V. Dolce and A. Forlano, Archaeological and Anthropological Sciences. 4 (Issue 2) (2012) 91-101. 


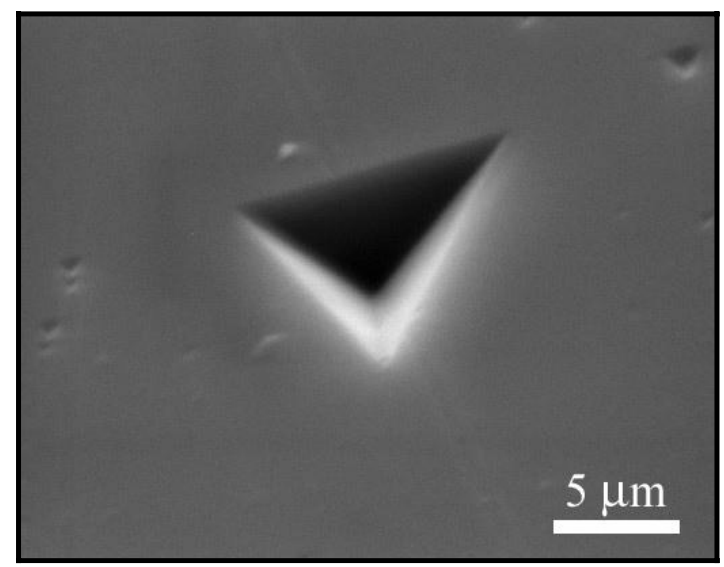

Figure 1: SEM micrograph of a single etch pit figure on aluminum surface.

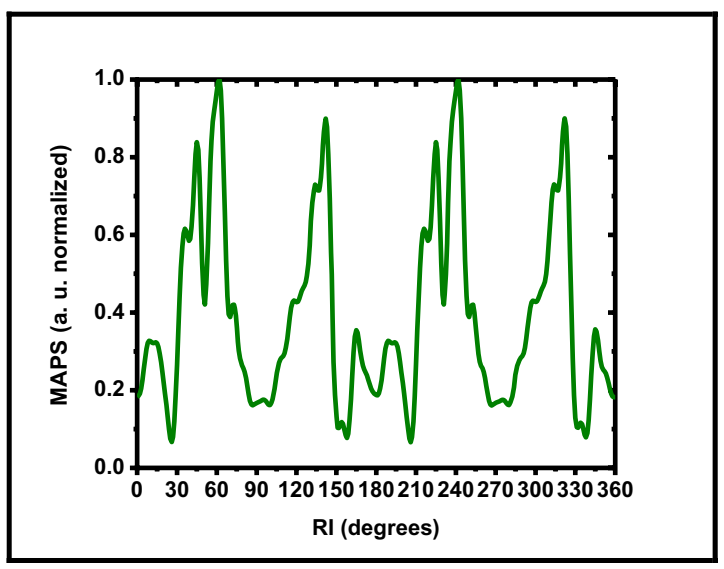

Figure 3: RIMAPS of the sketch of a single etch pit figure on aluminum surface.

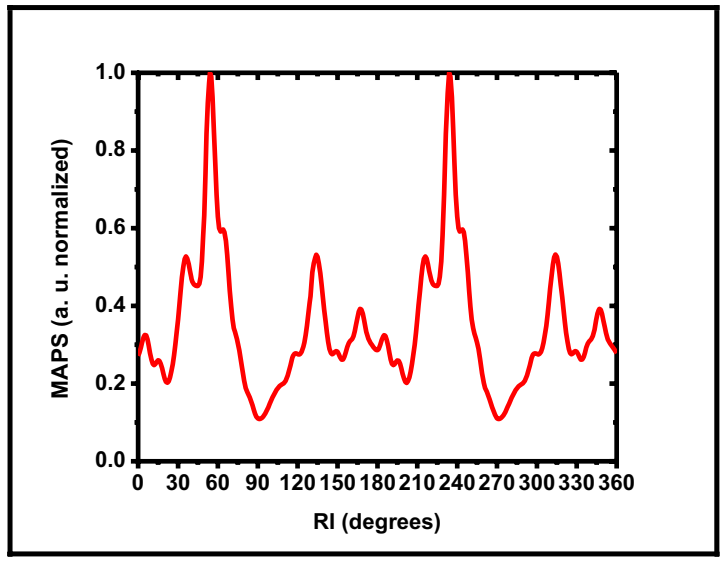

Figure 5: RIMAPS of the etch pit pattern on aluminum surface.

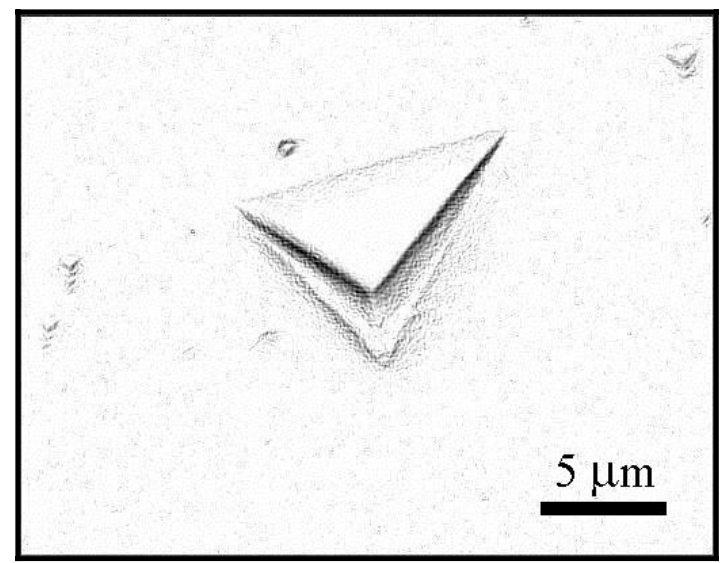

Figure 2: Sketch of the single etch pit figure on aluminum surface.

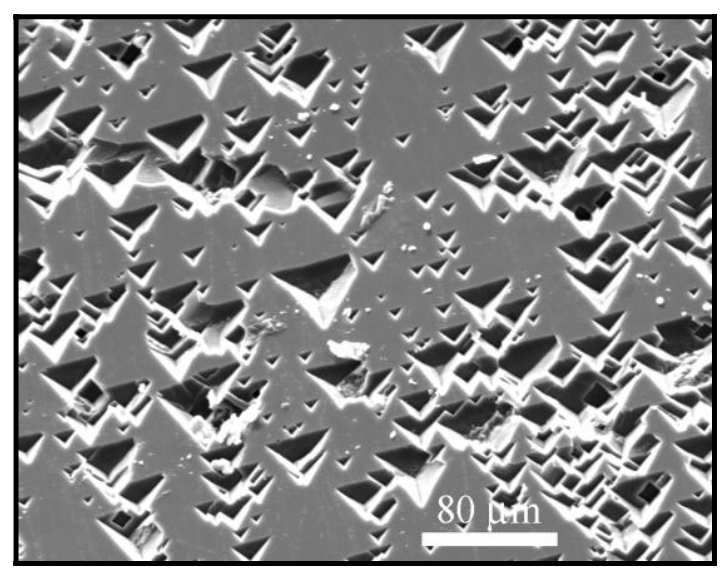

Figure 4: SEM micrograph of etch pit pattern on aluminum surface.

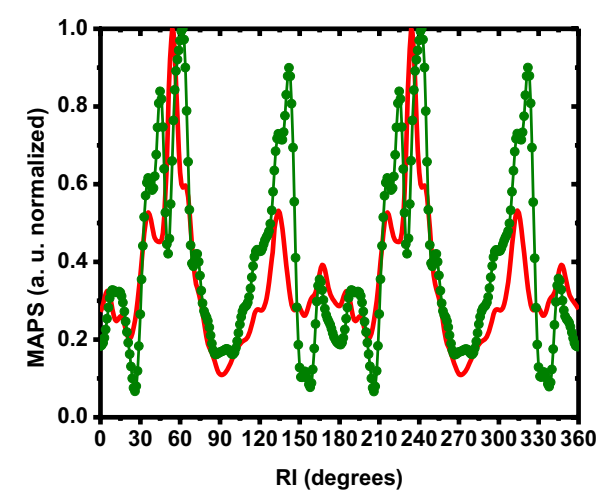

Figure 6: Comparison of RIMAPS spectra from the etch pit pattern and the single figure sketch $\longrightarrow \bullet-$ 